\title{
Total Flavonoids from Oroxylum indicum Induce Apoptosis via PI3K/Akt/PTEN Signaling Pathway in Liver Cancer
}

\author{
Nan-nan Li $\mathbb{D},{ }^{1}$ Xian-sheng Meng $\mathbb{D},{ }^{1,2,3}$ Wen-xiao Men, ${ }^{1}$ \\ Yong-rui Bao, ${ }^{1,2,3}$ and Shuai Wang ${ }^{1,2,3}$ \\ ${ }^{1}$ School of Pharmacy, Liaoning University of Traditional Chinese Medicine, Dalian 116600, China \\ ${ }^{2}$ Component Medicine Engineering Research Center of Liaoning Province, Dalian 116600, China \\ ${ }^{3}$ Liaoning Province Modern Chinese Medicine Research Engineering Laboratory, Dalian 116600, China \\ Correspondence should be addressed to Xian-sheng Meng; mxsvvv@163.com
}

Received 12 September 2017; Revised 12 January 2018; Accepted 16 January 2018; Published 15 February 2018

Academic Editor: Jae Youl Cho

Copyright (C) 2018 Nan-nan Li et al. This is an open access article distributed under the Creative Commons Attribution License, which permits unrestricted use, distribution, and reproduction in any medium, provided the original work is properly cited.

\begin{abstract}
Total flavonoids (TF), derived from the seeds of Oroxylum indicum (L.) Vent., possess many pharmacological functions. In the present study, $\mathrm{H}_{22}$-bearing mice and SMMC-7721 models were employed to evaluate the antitumor activity of TF and to and investigate its possible mechanisms both in vitro and in vivo. Cell viability was evaluated by MTT assay; cell apoptosis rate was analyzed via Annexin V-FITC/PI double staining by flow cytometer. Meanwhile, the expressions of apoptosis-related mRNA and proteins were evaluated by RT-PCR and Western blot analysis. The results revealed that TF could significantly inhibit the tumor growth, and the possible mechanism was related to the effect of inducing tumor cells apoptosis through PI3K/Akt/PTEN signaling pathway. This study has provided a theoretical basis for the further development and application of TF as antitumor drugs.
\end{abstract}

\section{Introduction}

Liver cancer, also known as hepatocellular carcinoma (HCC), and fewer special cases, which could include hepatoblastoma and angiosarcoma [1], is the 6th most common type of cancer in the world, with over 700,000 newly diagnosed patients per year. This burden of hepatocellular carcinoma will keep increasing till 2030 [2]. Moreover, smoking, alcohol abuse, and a history of diabetes mellitus may exacerbate the development of HCC [3]. Fluorouracil and tegafur are common, but not satisfactory, treatment of liver cancer, since they may lead to critical side effects and drug resistance [4]. A more effective therapy at present for liver cancer is transplantation, which, unfortunately, possesses a higher chance of metastasis and recurrence for such surgical resection [5]. Despite lack of scientific verification Traditional Chinese medicine (TCM) has been extensively used to treat different kinds of diseases due to its low toxicity, no drug resistance, and high efficiency $[6,7]$. Therefore the development of active ingredients derived from TCM, which possess better effectiveness and lower toxicity, has become an urgently necessary study for improving the treatment of liver cancer.
Oroxylum indicum (L.) Vent. has been extensively applied to treat cough, pertussis, pharyngitis, acute or chronic bronchitis, and other respiratory disorders. Current studies showed that TF are the main active components of Oroxylum indicum (L.) Vent. [8], which mainly contains oroxylin A, oroxylin $\mathrm{B}$, chrysin, chrysin-7-O- $\beta$-D glycosides, baicalein, and so on [9], with many pharmacological functions such as antiallergic [10], antioxidant [11], antimicrobial [12], antiinflammatory [13], and antitumor [14] functions. However, investigations on the antihepatoma effects of TF are limited, and the possible molecular mechanisms of the antitumor effects are still unrevealed. The present study was therefore aimed at investigating antitumor effect of TF and its possible molecular mechanisms.

\section{Materials and Methods}

2.1. Isolation and Purification of the TF. Oroxylum indicum was purchased from Tongrentang Pharmacy and authenticated by Professor Yanjun Zhai (School of Pharmacy, Liaoning University of Traditional Chinese Medicine, Liaoning, 
China) as dry seeds of Oroxylum indicum (L.) Vent. Using the best extraction and purification process which was got from the early research of the laboratory, Oroxylum indicum was extracted three times with 15 times the amount of $60 \%$ ethanol for $1 \mathrm{~h}$. HPD-100 resin was used, with the sample concentration of $0.1 \mathrm{~g} \cdot \mathrm{mL}^{-1}$, and HPD-100 resin with $5: 1$ of crude drugs BV column, after sufficient adsorption, with $5 \mathrm{BV}$ water to remove impurities and $10 \mathrm{BV} 60 \%$ ethanol elution, named TF, purity $\geq 90 \%$.

2.2. Tumor Cell Lines. Mouse $\mathrm{H}_{22}$ hepatocarcinoma cells $\left(\mathrm{H}_{22}\right)$ were obtained from the Shanghai Cell Bank, Chinese Academy of Science (Shanghai, China), and maintained in the peritoneal cavity of male ICR mice, used for further experiments.

2.3. Animals and Sample Preparation. Adult male ICR mice weighing 18-22 g were purchased from the experimental animal centre of Liaoning Changsheng Biological Technology Co., Ltd. Mice (except control group) were inoculated with $0.25 \mathrm{ml}$ of $5 \times 10^{5} / \mathrm{mL} \mathrm{H}_{22}$ cell suspension in the right armpit subcutaneously $[15,16]$. The animals were randomly divided into six groups, each consisting of twelve mice including blank group, control group, low-dose TF (TFL $50 \mathrm{mg} / \mathrm{kg}$ ), medium-dose TF (TFM $100 \mathrm{mg} / \mathrm{kg}$ ), and high-dose TF (TFH $200 \mathrm{mg} / \mathrm{kg}$ ) groups, and cyclophosphamide positive group $(50 \mathrm{mg} / \mathrm{kg})$. On the second day after the inoculation, the mice expect control and model groups were given the different doses of TF and cyclophosphamide intragastrically (blank and model control groups were given the same volume of normal saline) once daily consecutively for $12 \mathrm{~d}$; members of our research team observed mice daily for clinical signs of illness (e.g., weight loss and inactivity). Then mice were prohibited from any food but were allowed access to water freely for $12 \mathrm{~h}$ before the experiments; on the 12th day morning, taking drugs $1 \mathrm{~h}$ later, the body weight of mice was measured and the blood was collected by removing eyeball under sodium pentobarbital anesthesia (1\% sodium pentobarbital, $50 \mathrm{mg} / \mathrm{kg}$, intraperitoneal injection) in order to alleviate animal suffering. For collection of mouse tissues, mice were euthanized by carbon dioxide inhalation, and death confirmed by ascertaining cardiac and respiratory arrest. The tumors were removed and weighed; the tumor inhibition rate was calculated by the following formula: tumor inhibition rate $=(1-$ the average tumor weight medicine group/the average tumor weight control group) $\times$ $100 \%[17,18]$.

2.4. Histopathological Examination. For the histopathological analysis of tumor by HE straining method, tumor tissues of each group were fixed in $10 \%$ formalin solution for over $24 \mathrm{~h}$. After routine processing, the tumors were embedded in paraffin and cut at a thickness of $5 \mu \mathrm{m}$. Sections were stained with hematoxylin and eosin (H\&E) and subsequently examined using a light microscope for histopathological examination.

2.5. Measurement of BAX and Bcl-2 Level by ELISA Assay. The ELISA assay was performed to quantify tumor tissue levels of BAX and Bcl-2 according to the manufacturer's instructions. The absorbance was measured with a Spectra Max Plus microplate reader (Molecular Devices, CA, USA) at a wavelength of $450 \mathrm{~nm}$.

2.6. Cell Culture. The human hepatoma cell line SMMC7721 was purchased from the Shanghai Cell Bank, Chinese Academy of Science (Shanghai, China). This cell line was cultured in DMEM medium (Gibco, Grand Island, USA) supplemented with $1 \%$ penicillin/streptomycin (Gibco, Grand Island, USA) and 10\% FBS (fetal bovine serum) (Gibco, Grand Island, USA) at $37^{\circ} \mathrm{C}$ in a humidified incubator containing $5 \% \mathrm{CO}_{2}$.

2.7. MTT Colorimetric Assay. MTT assay was used to determine cell viability. Briefly, cells were plated in 96-well plates at a density of $1 \times 10^{4}$ per well. After overnight culture, different concentrations of TF $(0.2,0.6$, and $1.0 \mathrm{mg} / \mathrm{mL})$ were added to the wells and cells were incubated for 24,48 , and $72 \mathrm{~h}$. Then the culture medium was replaced with MTT (Sigma St. Louis, MO, USA) at final concentration of $2.5 \mathrm{mg} / \mathrm{mL}$, followed by $4 \mathrm{~h}$ incubation. Additionally, $150 \mu \mathrm{L}$ of Dimethyl sulfoxide (DMSO) (Sigma St. Louis, MO, USA) was added to each well. Absorbance was measured with a Spectra Max Plus microplate reader (Molecular Devices, CA, USA) at a wavelength of $492 \mathrm{~nm}$.

2.8. Cell Apoptosis. The apoptosis analyses by flow cytometer used FITC/PI double staining method; TF group cells including treated cells (cells were treated with TF for $48 \mathrm{~h}$ ) were collected and rinsed twice with cold PBS, mixed with $500 \mu \mathrm{L}$ of $1 \mathrm{x}$ binding buffer $\left(1 \times 10^{6} / \mathrm{mL}\right), 5 \mu \mathrm{L}$ Annexin V-FITC, and $5 \mu \mathrm{L}$ propidium iodide and incubated in the dark for $15 \mathrm{~min}$, and finally sent to the BD Accuir C6 flow cytometer (BD, USA) to analyze the cell apoptosis.

2.9. RNA Isolation and Real-Time Quantitative PCR Analysis. The human hepatoma cell line SMMC-7721 was plated in 6well plates at a density of $5 \times 10^{4}$ per well. After overnight culture, the TF at concentration of $1.0 \mathrm{mg} / \mathrm{mL}$ was added to the wells and cells were incubated for $48 \mathrm{~h}$. Total RNA of each group cells was extracted using TRIzol (Ambion, Texas, USA) according to the manufacturer's recommendations. Total RNA was reverse-transcribed using the TransScript First-Strand cDNA Synthesis SuperMix (TransGen Biotech, China). Quantitative real-time PCR was performed using TransStart Top Green Qpcr SuperMix (TransGen Biotech, China) with Piko Thermal Cycler 96-well system (Piko, Hawaii State, USA). Each sample was analyzed in triplicate. Relative levels of mRNA expression were normalized for $\beta$-actin mRNA expression and calculated according to the formula $2^{-(\Delta \mathrm{Ct} \text { sample- } \Delta \mathrm{Ct} \text { control })}$. Primers used were listed in Table 1. All primers were synthesized by Invitrogen, USA.

2.10. Western Blotting Analysis. The human hepatoma cell line SMMC-7721 was plated in 6-well plates at a density of $5 \times 10^{4}$ per well. After overnight culture, the TF at concentration of $1.0 \mathrm{mg} / \mathrm{mL}$ was added to the wells and cells 
TABLE 1: RT-PCR primers for each gene.

\begin{tabular}{lll}
\hline Gene & Forward primers & Reverse primers \\
\hline$\beta$-Actin & $5^{\prime}$-CACCCGCGAGTACAACCTTC-3' & $5^{\prime}$-CCCATACCCACCATCACACC-3' \\
Bax & $5^{\prime}$-TCATGGGCTGGACATTGGAC-3 & $5^{\prime}$-GAGACAGGGACACAGTCGC-3' \\
Bcl-2 & $5^{\prime}$-GTGAAGTCAACATGCCTGCC-3' & $5^{\prime}$-ACAGCCTGCAGCTTTGTTTC-3' \\
Caspase 3 & $5^{\prime}$-CCTGGTTCATCCAGTCGCTT-3' & $5^{\prime}$-TCTGTTGCCACCTTTCGGTT-3' $^{\prime}$ \\
PI3K & $5^{\prime}$-AACGAGAACGTGTGCCATTTG-3' & $5^{\prime}$-AGAGATTGGCATGCTGTCGAA-3' \\
p-Akt & $5^{\prime}$-CGAGGAGGAGGTGTATCA-3' & $5^{\prime}$-CGGTAAAGGCACGTTCGGTA-3 ${ }^{\prime}$ \\
PTEN & $5^{\prime}$-CCCAGTCAGAGGCGCTATG-3' & $5^{\prime}$-GGCAGACCACAAACTGAGGATT-3' \\
\hline
\end{tabular}

were incubated for $48 \mathrm{~h}$. Protein extracts were isolated from each group cells in using RIPA protein lysis buffer containing $1 \mathrm{mM}$ PMSF. Total protein was separated by $10 \%$ SDSPAGE, transferred with polyvinylidenedifluoride (PVDF) membrane, blocked in 5\% BSA (Solarbio, Beijing, China), and probed with appropriate primary antibodies against the target proteins. PTEN, PI3 Kinase p85, p-Akt, and $\beta$-actin antibodies were purchased from Cell Signaling (Beverly, MA, USA). These were followed by incubation with Goat Anti-Rabbit immunoglobulin (Ig) G ( $\mathrm{H}+\mathrm{L}$ ) (Protein tech, USA), and antigen-antibody complexes were visualized using the chemilucent ECL (TransGen Biotech, China) detection system. The densitometric analysis was conducted by using custom Image J-based software, as reported earlier $[19,20]$.

2.11. Statistics Analysis. Statistical analysis was performed using $t$-test or one-way ANOVA with GraphPad Prism 5. The $p$ values were considered statistically significant at $p<0.05$, and $p<0.01$ for very significant difference. All data are means \pm standard deviation (SD) for at least three separate experiments.

\section{Results}

3.1. Effect of TF on Tumor Growth in $\mathrm{H}_{22}$ Tumor-Bearing Mice. The histopathological examination was in order to evaluate the antitumor effect of TF; as shown in Figures 1(a)-1(e), the model group had large areas and density of tumor cells, which indicated that $\mathrm{H}_{22}$-bearing model was successfully established. The mice in positive and low-, medium-, and high-dose TF groups showed different degree of apoptosis areas compared to the model group.

The antitumor effect of TF on $\mathrm{H}_{22}$ tumor-bearing mice was showed in Figure 1(f). The results indicated that after the administration of TF for $12 \mathrm{~d}$ the average tumor weight was significantly decreased ( $p<0.01$ or $p<0.05$ ) compared to the model group.

3.2. Effect of TF on Tumor Tissue Levels of BAX and Bcl2 in $\mathrm{H}_{22}$ Tumor-Bearing Mice. We used the ELISA assay to investigate the effects of TF on the levels of BAX and Bcl2 in tumor tissues of $\mathrm{H}_{22}$ tumor-bearing mice. As shown in Figures 2(a)-2(b), the results indicated that the levels of BAX were significantly increased in the mice in mediumand high-dose $(p<0.01)$ compared with the control group, and Bcl-2 level of the mice in high-dose TF groups was significantly decreased when compared with the control group $(p<0.01)$.

3.3. Effect of TF on the Proliferation in the Human Hepatoma Cell Line SMMC-7721. In order to investigate the effect of TF on the proliferation of human hepatoma cell line SMMC7721 , we set up different concentrations of TF $(0.2,0.6$, and $1.0 \mathrm{mg} / \mathrm{mL}$ ) and then incubated cells for 24,48 , and $72 \mathrm{~h}$. The results indicated that TF could inhibit the proliferation of SMMC-7721 cells in a dose-dependent manner (Figure 3(d)).

3.4. Effect of TF on Apoptosis in the Human Hepatoma Cell Line SMMC-7721. The flow cytometry analysis showed that the percentage of apoptosis in TF group was $24.77 \pm 1.90 \%$; TF could significantly promote cell apoptosis when compared with the control group ( $p<0.01$ ), as shown in Figures $3(\mathrm{a})-3(\mathrm{c})$ and 3(e). We also determined the apoptosis of SMMC-7721 cell lines by RT-PCR analyses of Bax, Bcl-2, and Caspase 3. The results showed that the expression of Bcl2 was significantly decreased, and Bax and Caspase 3 were increased versus control group $\left({ }^{* *} p<0.01,{ }^{*} p<0.05\right)$ after cells were treated by TF (Figures 4(a)-4(b)). To summarize, these results suggested that TF inhibited cell proliferation by apoptosis.

3.5. The Effect of TF on p-Akt, PI3K, and PTEN mRNA Expression in SMMC-7721 Cells. To investigate the effect of TF on p-Akt, PI3K, and PTEN expression in SMMC7721 cells, we detected the expression by two-step RT-PCR analysis. As shown in Figures 4(c)-4(d), the expression of p-Akt and PI3K was significantly decreased, and PTEN increased versus control group $\left({ }^{* *} p<0.01,{ }^{*} p<0.05\right)$ after being treated by TF.

3.6. The Effect of TF on PI3K, p-Akt, and PTEN Protein Expression in SMMC-7721 Cells. Western blot analyses further confirmed whether lower levels of PTEN, p-Akt, and PI3K protein and higher levels of PTEN were observed in the TF treated cells. As shown in Figures 5(a)-5(b), p-Akt and PI3K protein expression were lower in TF treated cells versus control group $\left({ }^{*} p<0.05\right.$ or $\left.{ }^{* *} p<0.01\right)$. As shown in Figure 5, the expression of PTEN protein is significantly higher in TF treated cells versus the control group $\left({ }^{* *} p<\right.$ 0.01 ). 


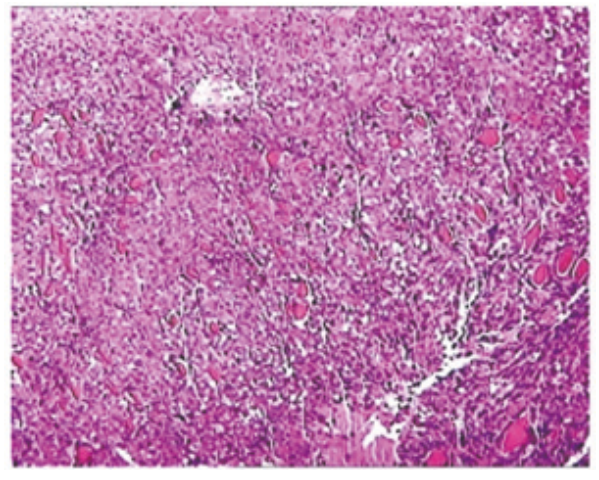

(a)

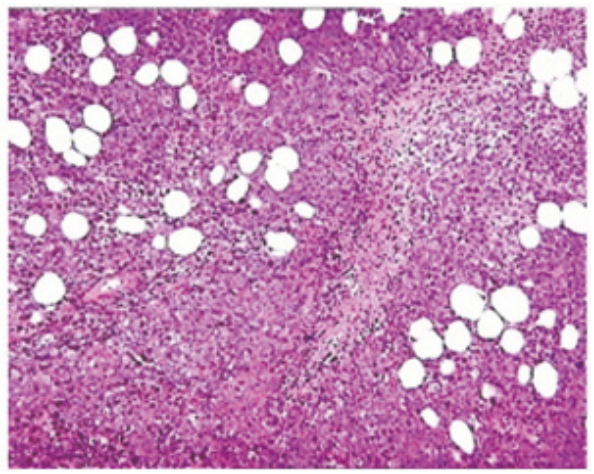

(c)

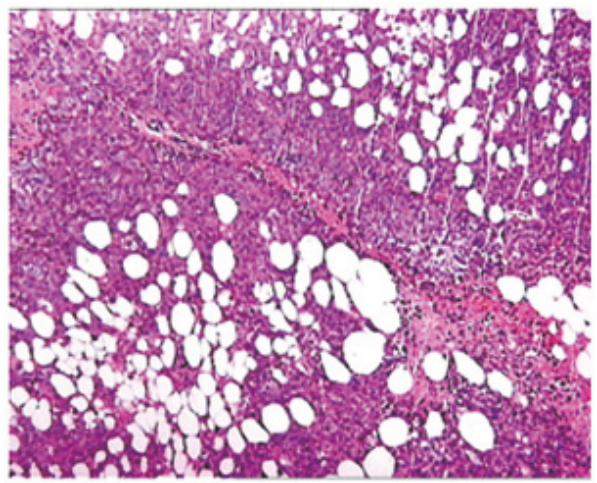

(e)

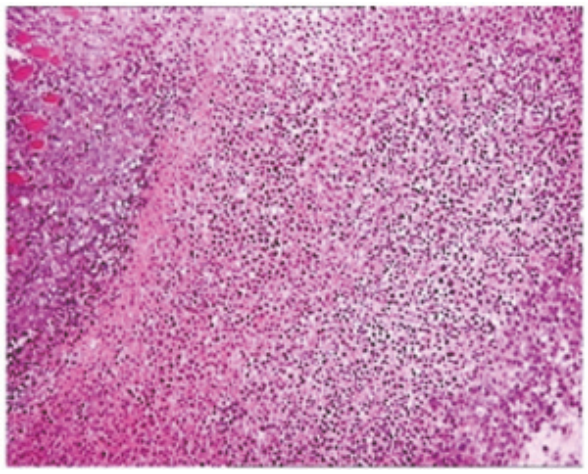

(b)

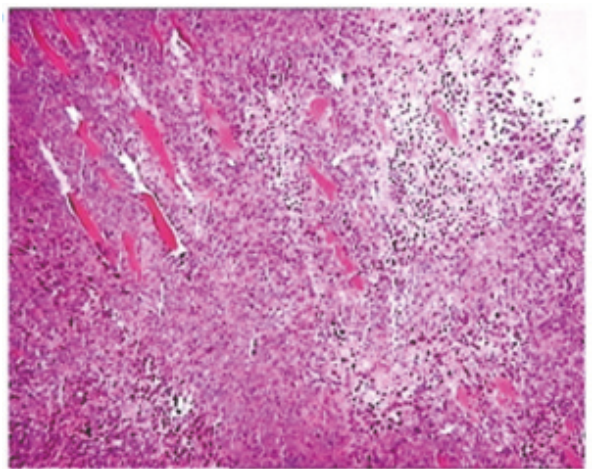

(d)

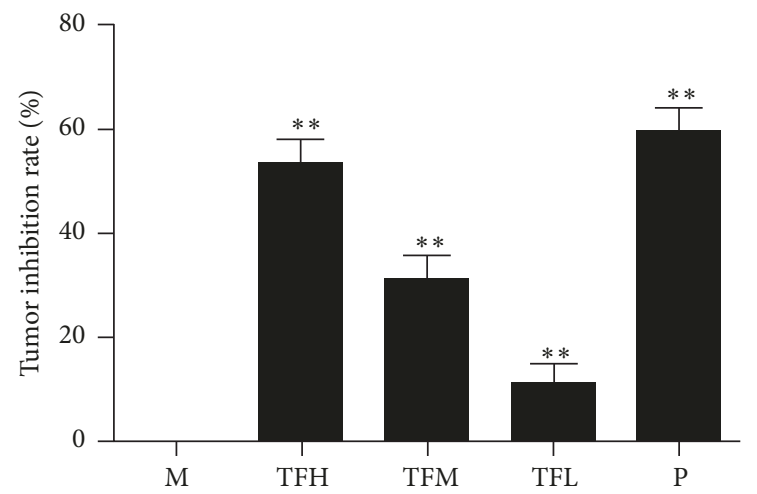

(f)

Figure 1: The effect of TF on tumor growth of $\mathrm{H}_{22}$ tumor-bearing mice. (a) Histopathological examination of control group, (b) positive group, (c) high-dose group, (d) medium-dose group, and (e) low-dose group. These tissues are stained by H\&E (200x). (f) The results of tumor inhibition rate, ${ }^{* *} p<0.01$.

\section{Discussion}

TF, as the active ingredients of Oroxylum indicum (L.) Vent., exert multiple physiological activities, including antitumor, antioxidation, antifatigue, anti-inflammation, and antimicrobial effect. In this study, the antitumor effect of TF and the possible molecular mechanism were investigated both in vitro and in vivo. From the results of histopathological examination (Figures 1(a)-1(e)), the high-, medium-, and low-dose TF group showed apoptosis in various degree versus control group which demonstrated that TF had good effect on inhibiting tumor cell proliferation and promoting their apoptosis. In addition, TF showed inhibitory effect on tumor growth in $\mathrm{H}_{22}$ tumor-bearing mice and SMMC-7721 cells, which exerted significant inhibition in a dose-dependent manner (Figures 1(f) and 3(d)). Result showed that TF had a good antihepatocellular carcinoma effect both in vitro and in vivo, which also indicated that $\mathrm{TF}$, as a potential antitumor agent, had the value of further research.

Apoptosis, a programmed cell death, is one of the main mechanisms of cell death in tumor treatment [21, 22]. It is regulated by many genes, including caspase and $\mathrm{Bcl}$ 2 family genes such as Bax and Bcl-2 [23-25]. The Bax 


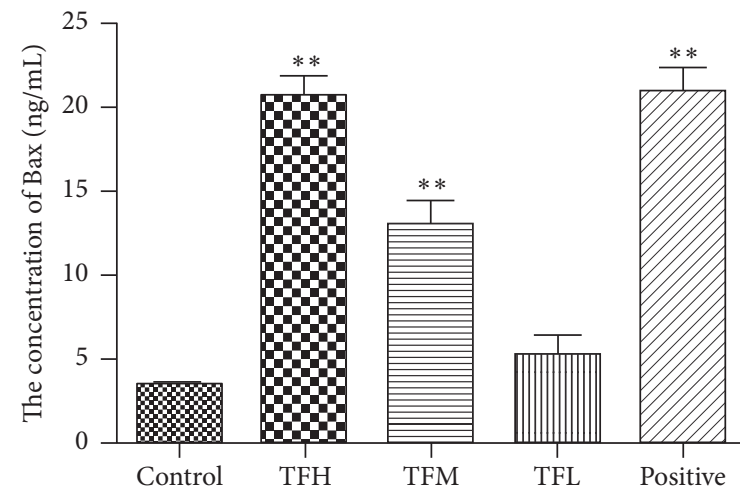

(a)

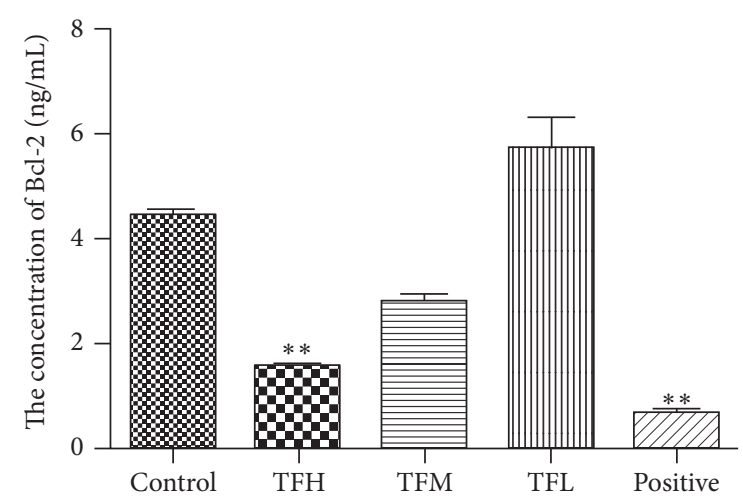

(b)

Figure 2: The levels of Bax and Bcl-2 in tumor tissue of $\mathrm{H}_{22}$ tumor-bearing mice. (a) The concentration of Bax. (b) The concentration of Bcl-2. All data were expressed as mean $\pm \mathrm{SD}, n=6 .{ }^{* *} p<0.01$.

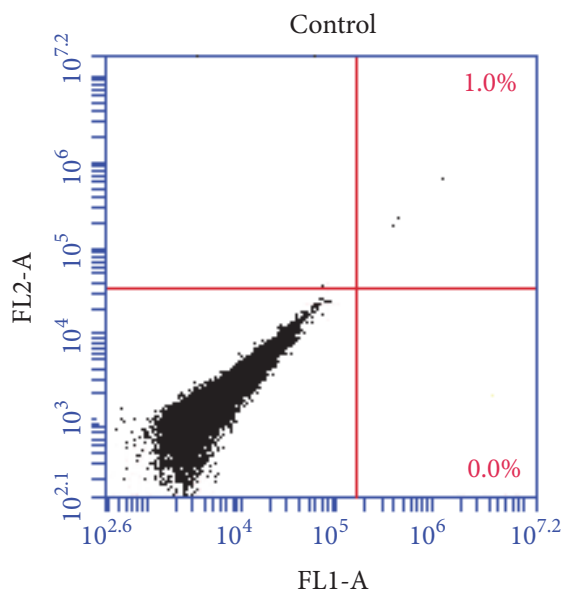

(a)

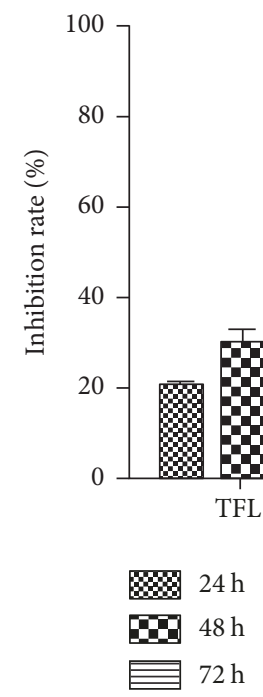

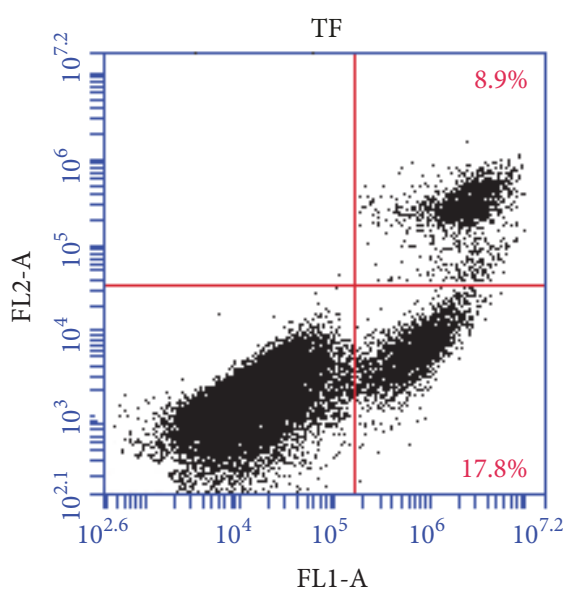

(b)

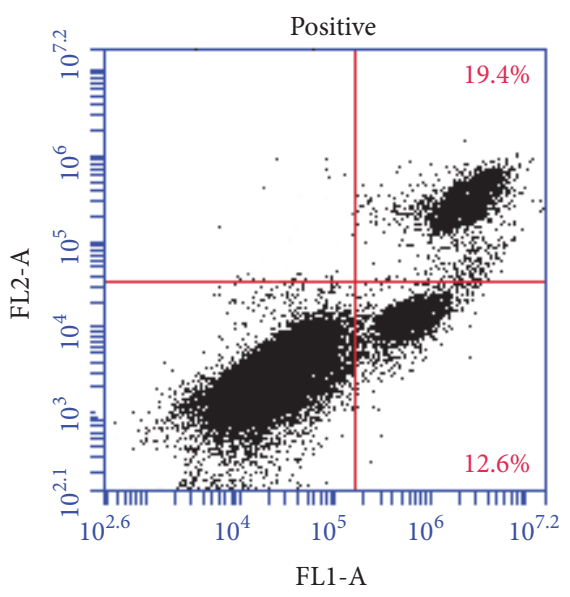

(c)

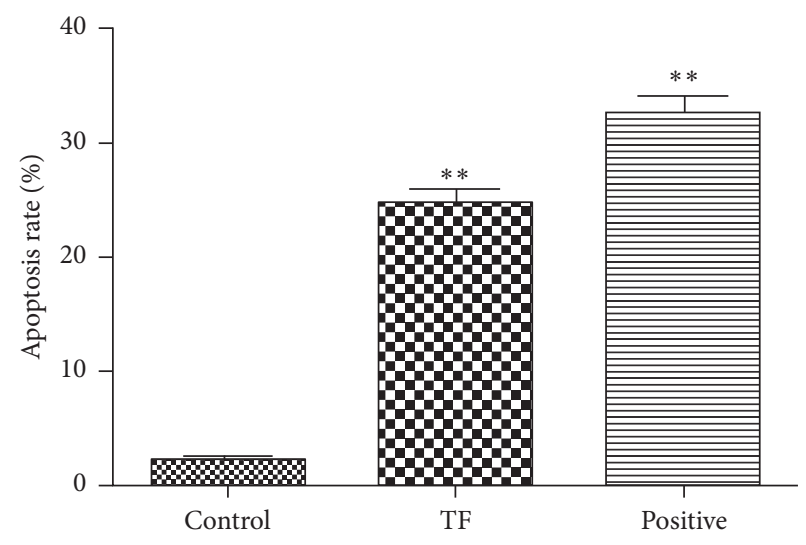

(e)

FIgURE 3: The effect of TF on apoptosis of SMMC-7721 cells. (a) Control group, (b) TF group, (c) positive group, and (e) statistical analysis results of cell apoptosis ratio by flow cytometry. ${ }^{* *} p<0.01$ versus control group; (d) TF inhibits the proliferation in the human hepatoma cell line SMMC-7721. Inhibition ratio of SMMC-7721 cells by TF with different concentrations $(0.2,0.6$, and $1.0 \mathrm{mg} / \mathrm{mL}) \mathrm{for} 24,48$, and $72 \mathrm{~h}$. All data were expressed as mean $\pm \mathrm{SD}, n=6$. 


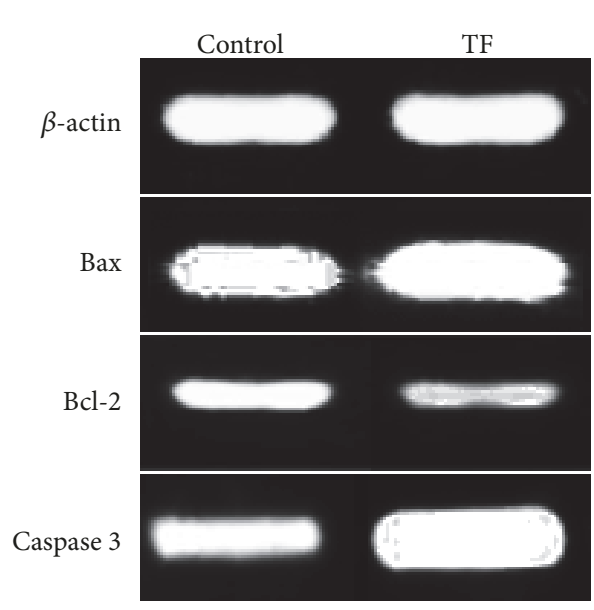

(a)

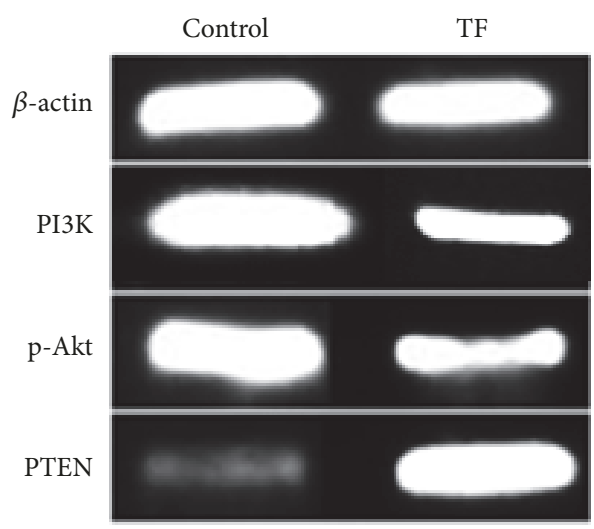

(c)

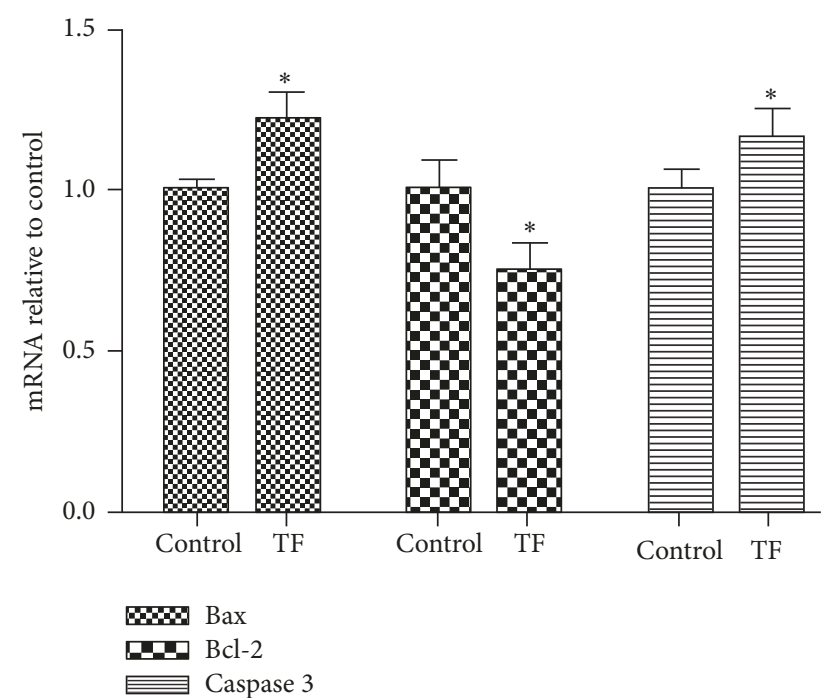

(b)

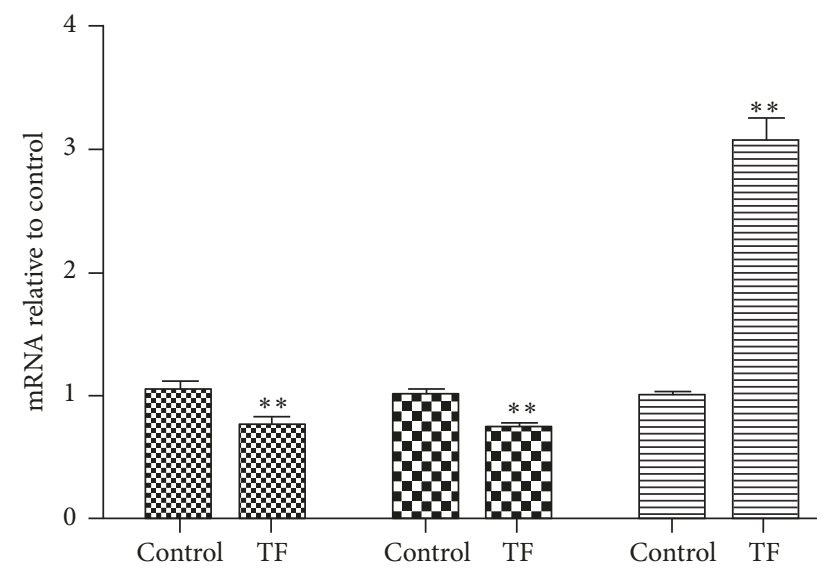

B\% PI3K

D.u. p-Akt

E PTEN

(d)

Figure 4: The expression of Bax, Bcl-2, Caspase 3, p-Akt, PI3K, and PTEN mRNA in SMMC-7721 cells. (a) The expression of Bax, Bcl-2, and Caspase 3 mRNA in SMMC-7721 cells and (b) Bax, Bcl-2, and Caspase 3 mRNA relative to control; (c) the expression of p-Akt, PI3K, and PTEN mRNA in SMMC-7721 cells and (d) PI3K, p-Akt, and PTEN mRNA relative to control. The levels of mRNAs were detected by RT-PCR and measured with $\beta$-actin as an internal reference. All data, repeated by three independent experiments, are presented as mean \pm SD. ${ }^{*} p<0.05$ and ${ }^{* *} p<0.01$ versus control group.

gene is a tumor suppressor gene, and $\mathrm{Bcl}-2$ is an antiapoptotic gene that antagonizes the function of Bax [26, 27]. Moreover, Caspase- 3 is the main process in the induction of apoptosis, since it is activated to regulate the caspasesignaling cascade and a downstream effector of apoptosis pathways [28]. In this study, the results of $\mathrm{HE}$ straining and flow cytometry showed that apoptosis occurred both in tumor tissues and in SMMC-7721 cells after TF intervention. We also determined the apoptosis by ELISA assay and RT-PCR method of BAX, Bcl-2, and Caspase 3 levels in tumor tissues of $\mathrm{H}_{22}$ tumor-bearing mice and SMMC7721 cells. These results suggested that, in either tumor tissues or SMMC-7721 cells, TF could downregulate Bcl-2 and upregulate Bax and Caspase 3 expressions. Therefore, the mechanism of antihepatocellular carcinoma of TF is related to induce apoptosis.

The PI3K/Akt/PTEN signaling pathways are crucial in many physiological and pathological conditions, such as cell proliferation, angiogenesis, and apoptosis [29, 30]. Previous studies showed that PI3K/Akt was frequently improperly activated in many kinds of human cancers [31], including hepatocellular carcinoma. It is closely associated with inhibiting the apoptotic cells and promoted the proliferation [32]. The PTEN is a key molecule downstream of the PI3K/Akt pathway and acts as a tumor suppressor by inhibiting cell growth and enhancing apoptosis $[33,34]$. In order to verify 


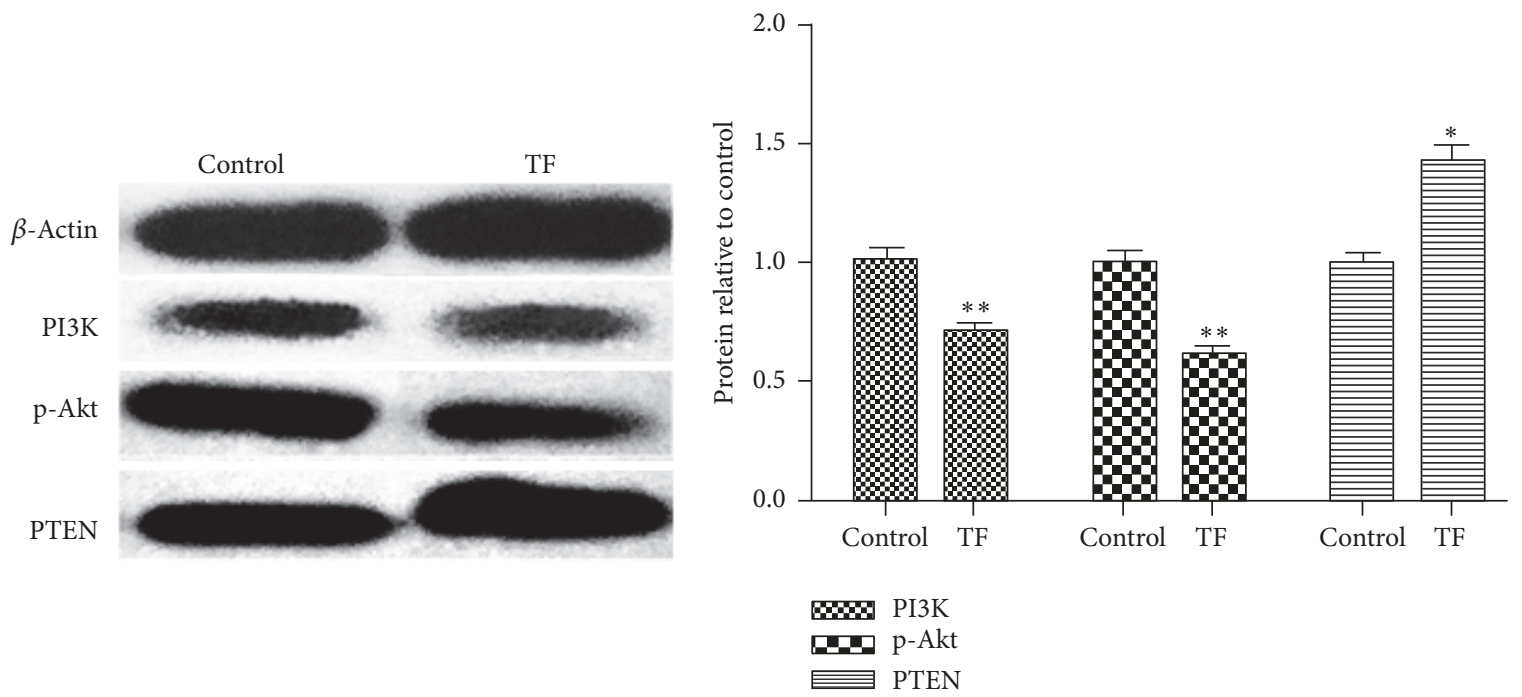

(a)

(b)

FIGURE 5: The expression of p-Akt, PI3K, and PTEN protein in SMMC-7721 cells. (a) Protein expression levels of PI3K, p-Akt, and PTEN in SMMC-7721 cells following TF treatment for $48 \mathrm{~h}$. (b) The expression of PI3K, p-Akt, and PTEN protein relative to control. All data, repeated by three independent experiments, are presented as mean \pm SD. ${ }^{*} p<0.05$ and ${ }^{* *} p<0.01$ versus control group.

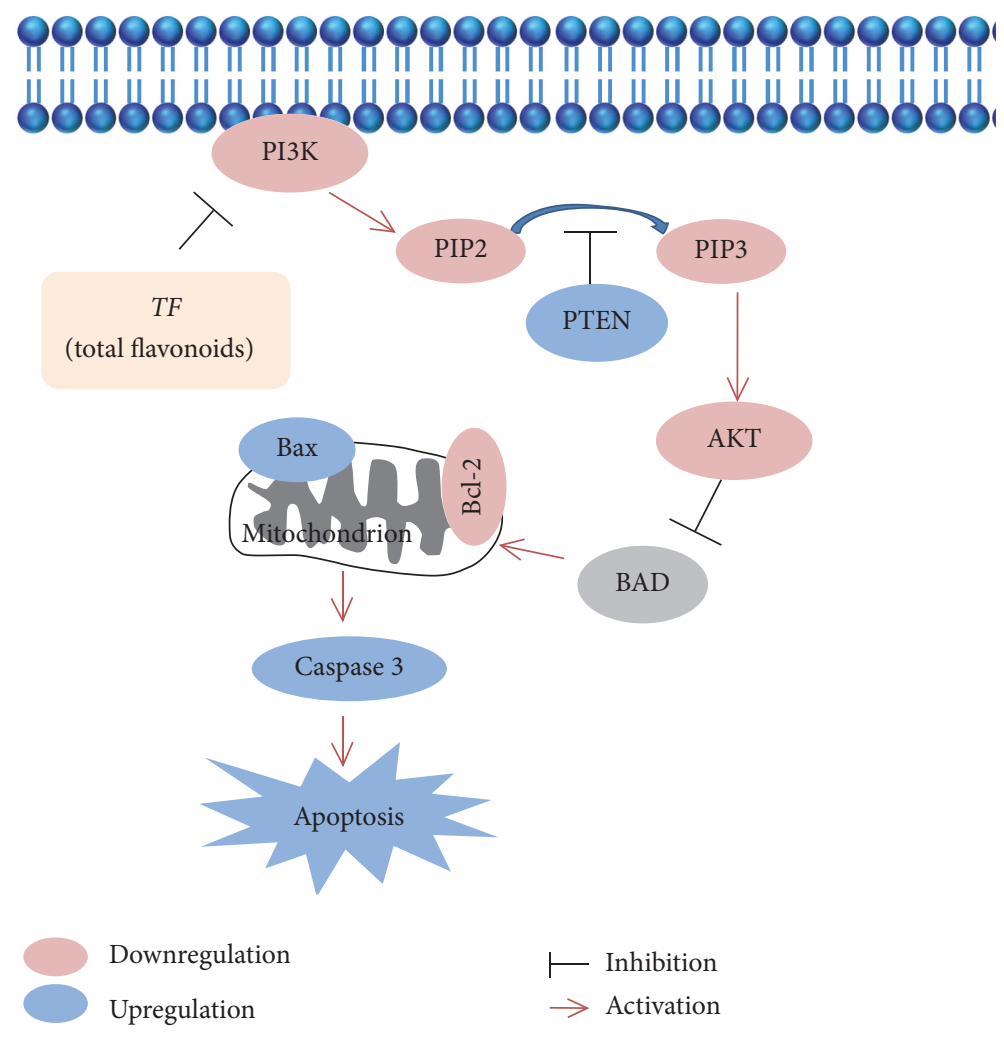

FIGURE 6: Anti-liver cancer mechanism of TF.

whether the antitumor effect of $\mathrm{TF}$ is related to the $\mathrm{PI} 3 \mathrm{~K} / \mathrm{Akt} / \mathrm{PTEN}$ pathway, and how to regulate related targets, PT-PCR and Western blot method were used. From the results of the study (Figures 4-5), TF could downregulate the expression of PI3K, p-Akt mRNA, and protein and upregulate the expression of PTEN. The results indicate that TF inhibited the PI3K/Akt/PTEN pathway and contributed to cellular apoptosis (as shown in Figure 6). In summary, TF acted as antitumor agent against liver cancer by promoting apoptosis via PI3K/Akt/PTEN signaling pathway. 


\section{Conclusion}

In conclusion, this research offered evidence that TF could significantly inhibit the growth of tumor in vitro and in vivo, possibly via inducing tumor cells apoptosis by PI3K/Akt/PTEN signaling pathway. Our research provided a scientific basis for the exploitation and application of total flavonoids in Oroxylum indicum (L.) Vent. as an antitumor agent, although further research will be needed before the clinical use.

\section{Conflicts of Interest}

The authors declare that there are no conflicts of interest in this manuscript.

\section{References}

[1] P. Bertuccio, C. Bosetti, F. Levi, A. Decarli, E. Negri, and C. La Vecchia, "A comparison of trends in mortality from primary liver cancer and intrahepatic cholangiocarcinoma in Europe," Annals of Oncology, vol. 24, no. 6, pp. 1667-1674, 2013.

[2] W. Ohishi, S. Fujiwara, J. B. Cologne et al., "Impact of radiation and hepatitis virus infection on risk of hepatocellular carcinoma," Hepatology, vol. 53, no. 4, pp. 1237-1245, 2011.

[3] H. B. El-Serag, T. Tran, and J. E. Everhart, "Diabetes increases the risk of chronic liver disease and hepatocellular carcinoma," Gastroenterology, vol. 126, no. 2, pp. 460-468, 2004.

[4] Q. Cai, J. Westin, K. Fu et al., "Accelerated therapeutic progress in diffuse large B cell lymphoma," Annals of Hematology, vol. 93, no. 4, pp. 541-556, 2014.

[5] Y. Zhou, C. Sui, B. Li et al., "Repeat hepatectomy for recurrent hepatocellular carcinoma: a local experience and a systematic review," World Journal of Surgical Oncology, vol. 8, article 55, 2010.

[6] W. Li, Y.-H. Tian, Y. Liu, Z. Wang, S. Tang, and J. Zhang, "Platycodin D exerts anti-tumor efficacy in $\mathrm{H}_{22}$ tumor-bearing mice via improving immune function and inducing apoptosis," Journal of Toxicological Sciences, vol. 41, no. 3, pp. 417-428, 2016.

[7] M. J. Hossen, M.-Y. Kim, J.-H. Kim, and J. Y. Cho, "Codonopsis lanceolata: a review of its therapeutic potentials," Phytotherapy Research, vol. 30, no. 3, pp. 347-356, 2016.

[8] J. Singh and P. Kakkar, "Oroxylin A, a constituent of Oroxylum indicum inhibits adipogenesis and induces apoptosis in 3T3-L1 cells," Phytomedicine, vol. 21, no. 12, pp. 1733-1741, 2014.

[9] T. H. Babu, K. Manjulatha, G. s. Kumar, A. Hymavathi, A. K. Tiwari, and M. Purohit, "Gastroprotective flavonoid constituents from Oroxylum indicum Vent," Bioorganic \& Medicinal Chemistry Letters, vol. 20, no. 1, pp. 117-120, 2010.

[10] A. Lee, S. Kang, S. Park, J. Huang, and D. Im, "Anti-allergic effect of oroxylin a from oroxylum indicum using in vivo and in vitro experiments," Biomolecules \& Therapeutics, vol. 24, no. 3, pp. 283-290, 2016.

[11] R.-Y. Yan, Y.-Y. Cao, C.-Y. Chen et al., "Antioxidant flavonoids from the seed of Oroxylum indicum," Fitoterapia, vol. 82, no. 6, pp. 841-848, 2011.

[12] D. S. Moirangthem, N. C. Talukdar, U. Bora, N. Kasoju, and R. K. Das, "Differential effects of Oroxylum indicum bark extracts: antioxidant, antimicrobial, cytotoxic and apoptotic study," Cytotechnology, vol. 65, no. 1, pp. 83-95, 2013.
[13] M. Rasadah, P. J. Houghton, A. Ramarr, and J. R. S. Hoult, "Antimicrobial and antiinflammatory activities of extractsand constituents of Oroxylum indicum (L.) Vent," Phytomedicine, vol. 5, pp. 375-381, 1998.

[14] P. Yang, S. Fu, Z. Cao et al., "Oroxin B selectively induces tumorsuppressive ER stress and concurrently inhibits tumor-adaptive ER stress in B-lymphoma cells for effective anti-lymphoma therapy," Toxicology and Applied Pharmacology, vol. 288, no. 2, pp. 269-279, 2015.

[15] S. Jiang, L. M. Qiu, Y. Q. Li, L. Li, X. Y. Wang, and Z. Liu, "Effects of Marsdeniatenacissima polysaccharide on the immune regulation and tumor growth in H22 tumor-bearing mice," Carbohydrate Polymers, vol. 137, pp. 52-58, 2016.

[16] S. Man, H. Chai, P. Qiu et al., “Turmeric enhancing antitumor effect of Rhizoma paridis saponins by influencing their metabolic profiling in tumors of $\mathrm{H} 22$ hepatocarcinoma mice," Pathology-Research and Practice, vol. 211, no. 12, pp. 948-954, 2015.

[17] K. F. Caleb, H. Y. Zhang, Y. Wang, J. J. Chen, X. Cao, and W. W. Deng, "Segetoside I, a plant-derived bisdesmosidicsaponin, induces apoptosis in human hepatoma cells in vitro and inhibits tumor growth in vivo," Pharmacological Research, vol. 110, pp. 101-110, 2016.

[18] Y. Shao, Y. Ni, J. Yang, X. Lin, J. Li, and L. Zhang, "Astaxanthin inhibits proliferation and induces apoptosis and cell cycle arrest of mice $\mathrm{H}_{22}$ hepatoma cells," Medical Science Monitor, vol. 22, pp. 2152-2160, 2016.

[19] M. J. Hossen, W. S. Yang, D. Kim, A. Aravinthan, J.-H. Kim, and J. Y. Cho, "Thymoquinone: an IRAK1 inhibitor with in vivo and in vitro anti-inflammatory activities," Scientific Reports, vol. 7, Article ID 42995, 2017.

[20] M. J. Hossen, J. Y. Cho, and D. Kim, "PDK1 in NF- $\kappa$ B signaling is a target of Xanthium strumarium methanolic extract-mediated anti-inflammatory activities," Journal of Ethnopharmacology, vol. 190, pp. 251-260, 2016.

[21] B. Fadeel and S. Orrenius, "Apoptosis: a basic biological phenomenon with wide-ranging implications in human disease," Journal of Internal Medicine, vol. 258, no. 6, pp. 479-517, 2005.

[22] X. X. Dong, J. Fu, X. B. Yin et al., "Induction of apoptosis in HepaRG cell line by aloe-emodin through generation of reactive oxygen species and the mitochondrial pathway," Cellular Physiology and Biochemistry, vol. 42, no. 2, pp. 685-696, 2017.

[23] R. Rosania, M. Varbanova, T. Wex et al., "Regulation of apoptosis is impaired in atrophic gastritis associated with gastric cancer," BMC Gastroenterology, vol. 17, no. 1, article 84, 2017.

[24] L. Gao, L. Wang, Z. Sun et al., "Morusin shows potent antitumor activity for human hepatocellular carcinoma in vitro and in vivo through apoptosis induction and angiogenesis inhibition," Drug Design, Development and Therapy, vol. 11, pp. 1789-1802, 2017.

[25] S. Xiang, T. Xiang, Q. Xiao, Y. Li, B. Shao, and T. Luo, "Zincfinger protein 545 is inactivated due to promoter methylation and functions as a tumor suppressor through the Wnt/ $\beta$ catenin, PI3K/AKT and MAPK/ERK signaling pathways in colorectal cancer," International Journal of Oncology, vol. 51, no. 3, pp. 801-811, 2017.

[26] M. Soleymaninejad, S. G. Joursaraei, F. Feizi, and I. J. Anarkooli, "The effects of lycopene and insulin on histological changes and the expression level of Bcl-2 family genes in the hippocampus of streptozotocin-induced diabetic rats," Journal of Diabetes Research, vol. 2017, Article ID 4650939, 9 pages, 2017.

[27] Y. P. Tang, F. M. Yu, G. Z. Zhang, Z. S. Yang, F. F. Huang, and G. F. Ding, "A purified serine protease from nereis virens and its 
impaction of apoptosis on human lung cancer cells," Molecules, vol. 22, no. 7, pp. 1123-1138, 2017.

[28] X.-C. Xie, Y. Cao, X. Yang, Q.-H. Xu, W. Wei, and M. Wang, "Relaxin attenuates contrast-induced human proximal tubular epithelial cell apoptosis by activation of the PI3K/Akt signaling pathway in vitro," BioMed Research International, vol. 2017, Article ID 2869405, 7 pages, 2017.

[29] J. Zhou, J. Ling, J. Song, Y. Wang, B. Feng, and F. Ping, "Interleukin 10 protects primary melanocyte by activation of Stat-3 and PI3K/Akt/NF- $\kappa$ B signaling pathways," Cytokine, vol. 83, pp. 275-281, 2016.

[30] L. N. He, J. Gubbins, Z. C. Peng, V. V. Medina, F. Fei, and K. J. Asahina, "Activation of hepatic stellate cell in Ptennull liver injury model," Fibrogenesis \& Tissue Repair, vol. 9, pp. 8-20, 2016.

[31] B. Li, Z. Wang, Y. Zhong, J. Lan, X. Li, and H. Lin, "CCR9CCL25 interaction suppresses apoptosis of lung cancer cells by activating the PI3K/Akt pathway," Medical Oncology, vol. 32, no. 3, pp. 66-75, 2015.

[32] L. Zheng, W. Gong, P. Liang et al., "Effects of AFP-activated PI3K/Akt signaling pathway on cell proliferation of liver cancer," Tumor Biology, vol. 35, no. 5, pp. 4095-4099, 2014.

[33] P. Camillo, P. Chiara, and M. Alessandra, "Targeting PI3K/Akt/ mTOR signaling in cancer," Frontiers in Oncology, vol. 4, pp. 6474, 2014.

[34] N. McCabe, S. M. Walker, and S. D. Kennedy, "When the guardian becomes the enemy: targeting ATM in PTEN-deficient cancers," Molecular Cellurar Oncology, vol. 3, Article ID e1053595, 2016. 


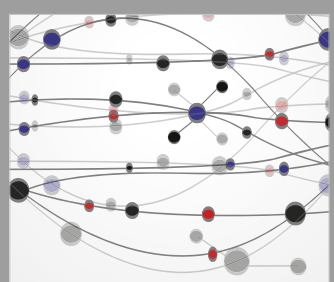

The Scientific World Journal
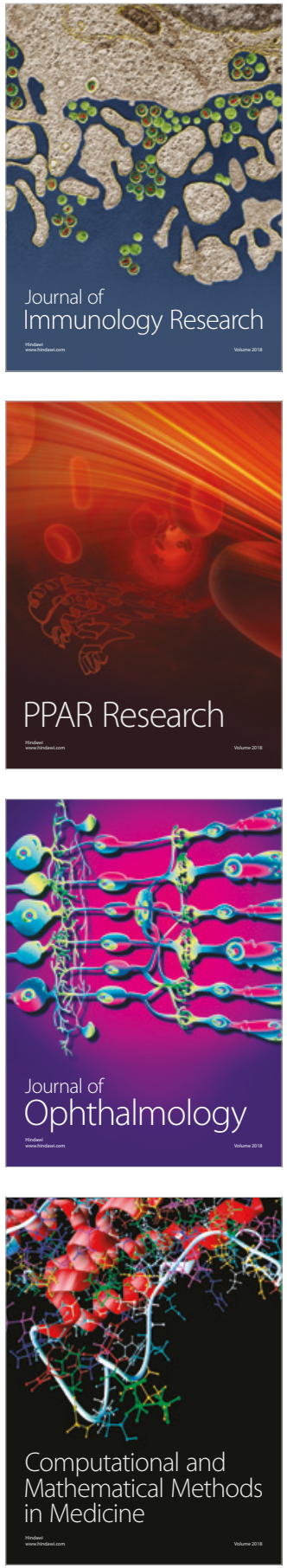

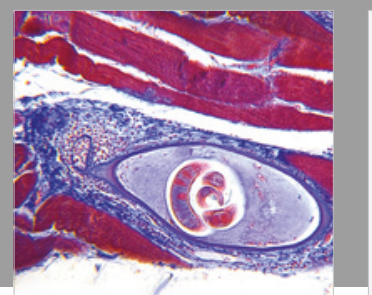

Gastroenterology Research and Practice

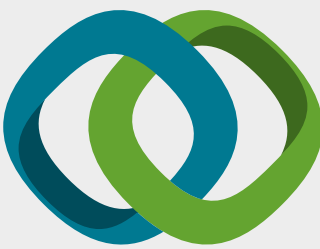

\section{Hindawi}

Submit your manuscripts at

www.hindawi.com
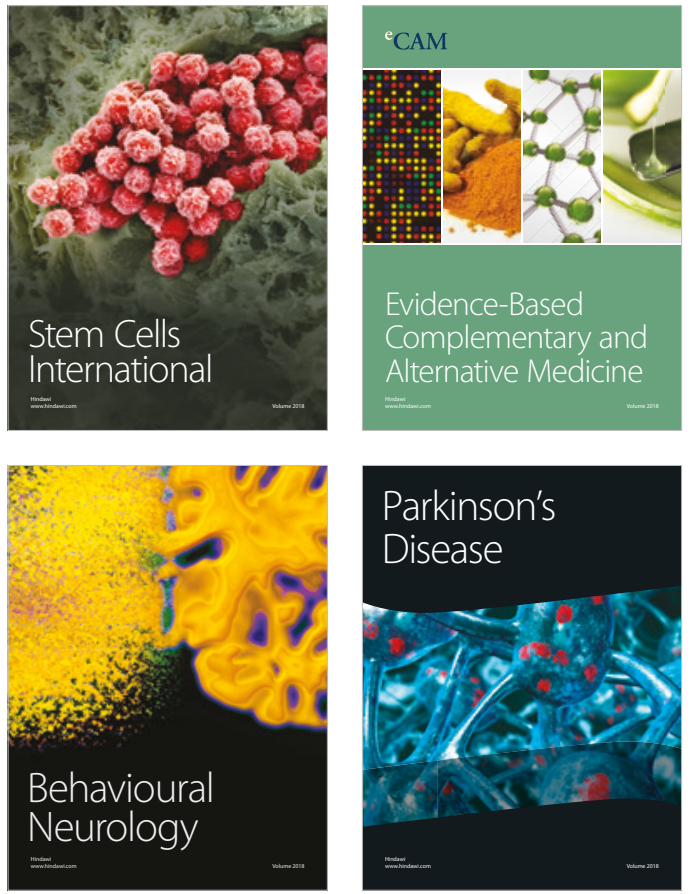

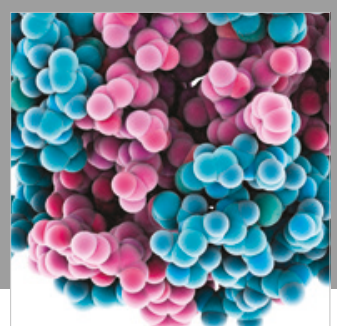

ournal of

Diabetes Research

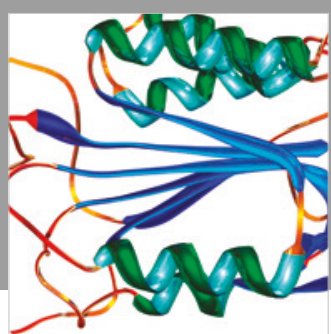

Disease Markers
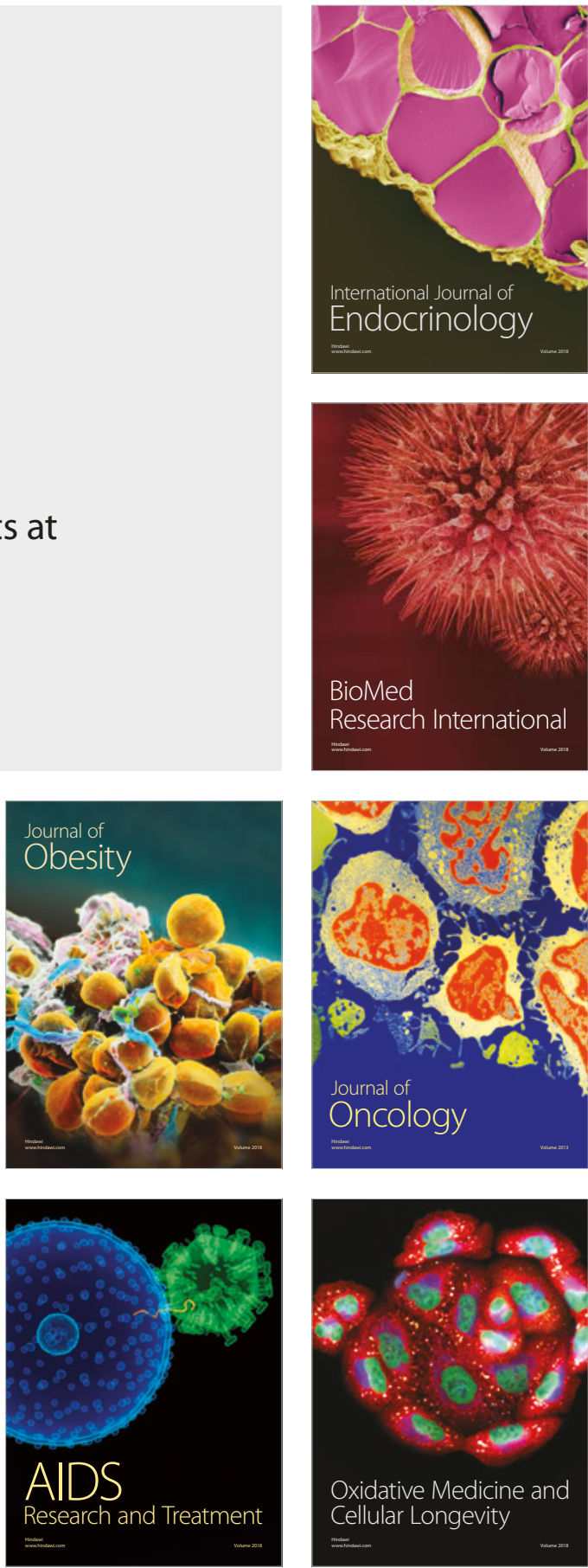